\title{
Configurations for short period rf undulators
}

\author{
S. V. Kuzikov, ${ }^{1} *$ Y. Jiang, ${ }^{2}$ T. C. Marshall, ${ }^{3,4}$ G. V. Sotnikov, ${ }^{5}$ and J. L. Hirshfield ${ }^{2,3}$ \\ ${ }^{1}$ Institute of Applied Physics, Nizhny Novgorod, Russia \\ ${ }^{2}$ Yale University, New Haven, Connecticut 06511, USA \\ ${ }^{3}$ Omega-P, Inc., Yale University, New Haven, Connecticut 06510, USA \\ ${ }^{4}$ Columbia University, New York, New York 10027, USA \\ ${ }^{5}$ Kharkov Institute of Physics and Technology, Kharkov, Ukraine \\ (Received 16 January 2013; published 19 July 2013)
}

\begin{abstract}
Several configurations for rf undulators energized at millimeter wavelengths and designed to produce coherent nanometer radiation from sub-GeV electron beams are analyzed and compared with one another. These configurations include a traveling-wave resonant ring, a standing wave resonator, and a resonator operating close to cutoff.
\end{abstract}

DOI: $10.1103 /$ PhysRevSTAB.16.070701

PACS numbers: 41.75.Lx, 41.60.Bq

\section{INTRODUCTION}

A conventional undulator comprising a static magnetic field typically has a period of a few centimeters. So in order to produce free-electron laser (FEL) radiation at $\mathrm{nm}$ wavelengths, a $1-2 \mathrm{GeV}$ electron beam is required. It should therefore have strong appeal for a nm-wavelength light source if, instead, a beam of several times less energy were required through use of an undulator with a period of under $1 \mathrm{~cm}$. This appealing scenario might be realized through use of an $\mathrm{rf}$ undulator, which could have a mm-scale period plus twice the frequency up-shift of a static undulator $[1,2]$. Further, an rf undulator can have a $\mathrm{cm}$-scale aperture to avoid electron beam interception, and can be immune to radiation damage that might affect exotic magnetic materials or superconducting coils [3]. An rf undulator could also provide fast dynamic control of polarization, wavelength, and undulator $K$ parameter, and incorporate field tapering to compensate for radiative energy loss from the wiggling particles [4,5]. For a static undulator, the undulator parameter $K$ in practical units is given by $K=93.4 H_{\perp}[\mathrm{T}] \lambda_{u}[\mathrm{~m}]$, where $H_{\perp}$ and $\lambda_{u}$ are the transverse magnetic field and period of the undulator.

The earliest demonstration of an $\mathrm{rf}$ undulator was by Shintake et al., about 30 years ago [6]. That device employed a meter-long $\mathrm{TE}_{1,0,19}$-mode cavity that used a rectangular ridged waveguide driven with $300 \mathrm{~kW}$ of rf power at $2856 \mathrm{MHz}$, but it had a relatively small $K$ parameter $(K \approx 0.2)$. In Denisov's experiment, carried out at $10 \mathrm{GHz}$, the $K$ parameter was increased to be close to unity through the use of MW-level rf power [7]. Recently Tantawi et al.

\footnotetext{
*kuzikov@appl.sci-nnov.ru

Published by the American Physical Society under the terms of the Creative Commons Attribution 3.0 License. Further distribution of this work must maintain attribution to the author(s) and the published article's title, journal citation, and DOI.
}

reported the observation of the optical radiation $(800 \mathrm{~nm}$ wavelength) from an rf undulator fed by $48 \mathrm{MW}$ power in X-band [8].

To have rf undulator magnetic fields as high as those produced by available permanent magnets or superconducting coils $(\sim 1 \mathrm{~T})$ and corresponding $K$ values close to unity, it would require GW-level propagating $\mathrm{cm}$ - or $\mathrm{mm}$-wavelength $\mathrm{rf}$ power in a waveguide of $\sim 1 \mathrm{~cm}$ radius [9]. As an example, Pelligrini [9] described an X-band undulator that used a square waveguide operating in a rotating TE mode that would require rf powers as high as $400 \mathrm{MW}$ to provide an undulator parameter $K=0.4$ with an undulator period $\lambda_{u}=1.45 \mathrm{~cm}$. Since this paper concerns undulators with shorter wavelengths, and, since the power of available phase-coherent $\mathrm{cm}$ - and $\mathrm{mm}$-wave rf amplifiers such as the magnicon or relativistic gyroklystron is limited to several tens of megawatts $[10,11]$, it thus follows that to obtain $T$-level undulator fields will require the use of high $Q \mathrm{rf}$ resonant cavities.

The design of cavities for high-power mm-wavelength radiation requires that attention be paid to $\mathrm{rf}$ breakdown and pulsed surface heating limits, in addition to Ohmic wall losses. Furthermore, in rf undulators, beam dynamics issues that lead to emittance growth and associated distortions of the radiation spectrum must be investigated. These issues arise because of peculiarities for $\mathrm{rf}$ undulators that are not found with static magnetic undulators. One such peculiarity is a defocusing ponderomotive (Miller) force on beam electrons caused by transverse field gradients $[12,13]$, while another arises from the standing wave nature of cavity fields that are superpositions of waves that counterpropagate and copropagate with respect to the electron beam $[14,15]$.

This paper considers a traveling-wave resonator, a standing wave resonator, and a resonator operating close to cutoff-all as candidates to confront practical rf undulator limitations. Detailed designs of these mentioned configurations will be described in a future paper. 


\section{II. $K$ PARAMETER FOR RF UNDULATORS}

Parameters of undulators driven by periodic static magnetic fields are thoroughly described in the FEL literature $[1,2]$. In an rf undulator, where relativistic electrons with normalized longitudinal velocity $\beta_{z}=v_{z} / c \approx 1$ along $z$ oscillate in periodic microwave electric and magnetic fields, significant differences exist, as compared with static magnetic undulators. In general, $\mathrm{rf}$ fields in a resonator are both counterpropagating and copropagating, relative to motion of the electron beam. The counterpropagating waveguide wave is responsible for the desired highfrequency wiggle motion of electrons with a period $\lambda_{u}$, where

$$
\lambda_{u}=\frac{2 \pi}{k_{u}}=\frac{2 \pi}{h+k / \beta_{z 0}} \cong \frac{2 \pi}{h+k},
$$

where $k_{u}$ is the wave number in the waveguide, $h=2 \pi / \lambda_{g}$ is the propagation constant of a wave in a waveguide at angular frequency $\omega, k=\omega / c=2 \pi / \lambda$ is the vacuum wave number, and $\beta_{z 0}$ is the average normalized longitudinal velocity. Such wiggle motion induces resonant scattering into a light wave at angular frequency $\omega_{s}$ propagating along the $z$ axis in accordance with the well-known resonance condition [14],

$$
\frac{\omega_{s}}{c}=\gamma_{z 0}^{2}\left(1+\beta_{z 0}\right)\left(k+h \beta_{z 0}\right),
$$

where $\gamma_{z 0}=\left(1-\beta_{z 0}^{2}\right)^{-1 / 2}$. It follows from Eq. (2) that the frequency up-conversion $\omega_{s} / \omega$ for a paraxial waveguide wave close to a plane wave in an $\mathrm{rf}$ undulator $(h \approx k)$ is roughly proportional to $4 \gamma_{z 0}^{2}$, instead of roughly proportional to $2 \gamma_{z 0}^{2}$ as in a DC undulator.

One of the most important parameters is the undulator parameter $K$ that determines the magnitude of the wiggling motion. In an undulator consisting of DC magnets the $K$ parameter is written in terms of the perpendicular magnetic field and undulator period as

$$
K=\frac{e H_{\perp} \lambda_{u}}{2 \pi m c^{2}},
$$

in centimeter-gram-second (CGS) units, and where $e$ and $m$ are the charge and mass of the electron, respectively. The equations in this paper will use CGS units to simplify the expressions and emphasize the equivalent effect from both electric field and magnetic field components of the microwave to the electron wiggling motion.

Unlike the DC undulator, in the rf undulator both transverse electric and magnetic fields cause electron wiggling motion. In Appendix A, equations for the $K$ parameter in an rf undulator are derived using simple geometrical considerations; while in the next section the same equations are derived using a perturbation method to solve for particle motion in assigned electromagnetic fields. In numerous papers devoted to undulators consisting of DC magnets (for example in Ref. [2]), the formula for the output light frequency Eq. (2) is frequently rearranged using the fact that in a static magnetic field the total particle energy $m c^{2} \gamma_{0}$ is constant (neglecting radiation losses) and equal to its initial value upon entering the undulator. Thus, one may write

$$
\lambda_{s} \equiv \frac{2 \pi c}{\omega_{s}}=\frac{\lambda_{u}\left(1+K^{2} / 2\right)}{2 \gamma_{0}^{2}},
$$

where $\lambda_{s}$ is the optical wavelength. As it will be shown in Sec. III [see Eq. (33)] and Appendix A this formula is still valid in some important cases for if undulators, although $\gamma$ is not strictly a constant. Under the assumption that $h \approx k$ (in the paraxial wave approximation, $\lambda_{u} \approx \lambda / 2$ ) we find in place of Eq. (4) the result

$$
\lambda_{s} \equiv \frac{2 \pi c}{\omega_{s}}=\frac{\lambda\left(1+K^{2} / 2\right)}{4 \gamma_{0}^{2}} .
$$

Comparison of Eqs. (4) and (5) shows that one might reach 2 times shorter wavelength FEL radiation for an rf undulator whose wavelength equals the period in a DC magnetic undulator.

Apart from the $K$ parameter, another important parameter is the gain length $L_{g}$. The gain length scales the distance over which radiation power and longitudinal beam microbunching grow up in an undulator in the regime of selfamplified spontaneous emission (SASE). In FEL the optimal length of each undulator segment is dependent on many factors, including beta function (related to quadrupole focal length) and beam line filling factor, but the undulator length should scale as the gain length.

In particular, the $1 \mathrm{D}$ gain length $L_{g}$ for a planar monoenergetic beam moving in a linearly polarized wave scales inversely with the dimensionless Pierce parameter $\rho$, namely [2],

$$
L_{g}=\frac{\lambda_{u}}{4 \pi \sqrt{3} \rho}
$$

where

$$
\rho=\left[\frac{\eta K^{2}[J J]^{2} \lambda_{u}^{2}}{\gamma^{3} \lambda_{s}^{2}}\right]^{1 / 3} .
$$

In Eq. (7), the dimensionless parameter $\eta$ describes the electron current density and beam size, depends on beam parameters only and not on the undulator parameter $K$, i.e.,

$$
\eta=\frac{\lambda_{s}^{2}}{64 \pi^{2} \sigma_{x}^{2}} \frac{I_{e}}{I_{A}},
$$

where $I_{e}$ is the peak electron current, $I_{A}$ is the Alfven current $\left(I_{A}=17 \mathrm{kA}\right), \sigma_{x}$ is the rms transverse size of the beam, $[J J]=J_{0}(\zeta)-J_{1}(\zeta)$ with $J_{0}$ and $J_{1}$ the zeroth- and first-order Bessel functions with argument. For example, if one takes $\lambda_{s}=2 \mathrm{~nm}, \sigma_{x}=0.1 \mathrm{~mm}$, and $I_{e}=1 \mathrm{kA}$, then $\eta \approx 10^{-14}$. Equations (5)-(7) allow one to write the scaling law for an undulator period in terms of output optical wavelength and undulator parameter in the form 


$$
\lambda_{u}^{5}=\left(8 \sqrt{3} \pi L_{g}\right)^{6} \eta^{2} \lambda_{s}^{-1}\left(\frac{K^{4 / 3}}{2+K^{2}}\right)^{3}[J J]^{4} .
$$

For optimizing an rf undulator design based on a typical FEL user's preference for a given optical wavelength $\lambda_{s}$, with a fixed injection beam parameter $\eta$, and overall length scaling as $L_{g}$, the first-order considerations lead to the approximate scaling law,

$$
K \sim \lambda^{5 / 4} \lambda_{s}^{1 / 4}
$$

where a far-from-cutoff $\mathrm{rf}$ wave $\left(\lambda_{u} \approx \lambda / 2\right)$ was assumed, and it was taken into account that for small values of $K$, $[J J] \approx 1$. Equation $(10)$ suggests that it may not be inaccurate to take the $K$ parameter as scaling almost linearly with rf wavelength. Naturally, output powers of electron rf sources decrease with wavelength (the particular law depends on source type). Equation (10) shows how to find a compromise between possible rf wavelength and necessary output power (proportional to $K^{2}$ ), when one chooses an appropriate rf source for undulator.

In undulators based on DC magnets with a period of several centimeters, the desired undulator parameter is typically slightly greater than unity. But the above analysis suggests that, in an rf undulator fed by $\sim 1 \mathrm{~cm}$ wavelength power, the required $K$ parameter could be several times less, i.e., less than unity.

Results of calculations for the dependence of $K$ on undulator period $\lambda_{u}$ obtained using the exact formula Eq. (9) for $\lambda_{s}=2 \mathrm{~nm}, L_{g}=1 \mathrm{~m}$ and for different beam

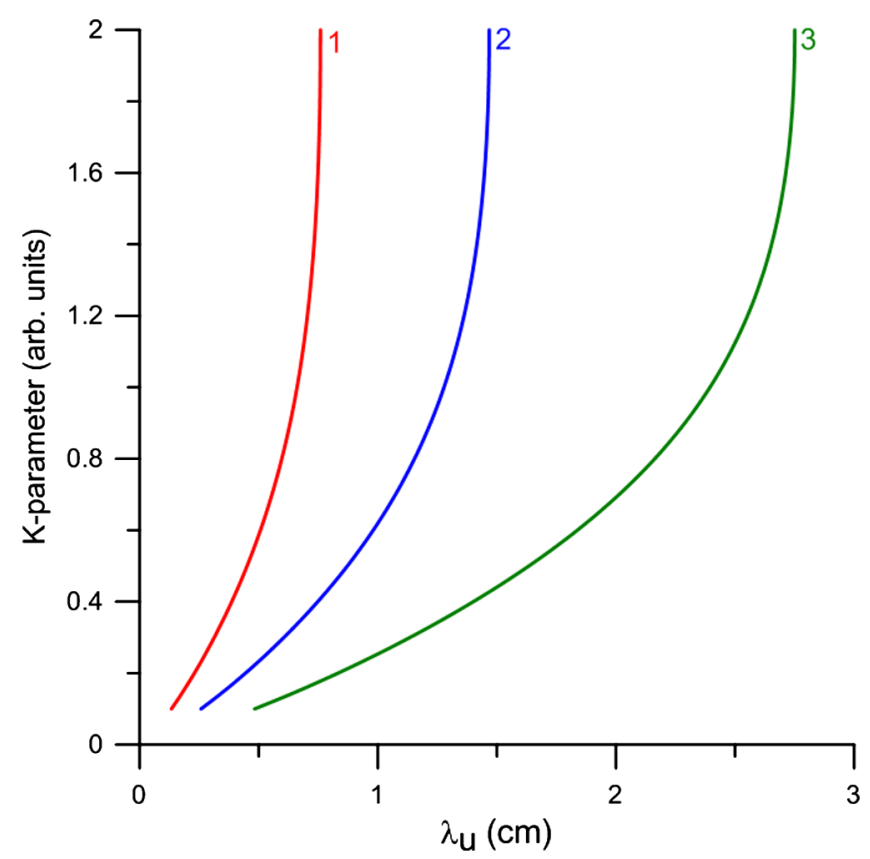

FIG. 1. Parameter $K$ versus undulator period $\lambda_{u}$ : curve $1-$ $\eta=10^{-14}$; curve $2-\eta=5 \times 10^{-14}$; and curve $3-\eta=2.5 \times$ $10^{-13}$. The higher $\eta$ indicates the higher electron current density and the smaller beam size. parameters $\eta$ are plotted in Fig. 1. The curves show that the undulator parameter $K$ drops dramatically as the undulator period $\lambda_{u}$ is reduced, for fixed $\lambda_{s}$ and $L_{g}$. Near small $K$ values the undulator parameter almost linearly depends on wavelength.

\section{GENERAL PROPERTIES OF RF UNDULATORS}

Possible configurations for an rf undulator can be based on either a traveling-wave cavity [9], or a standing wave cavity far or near to cutoff [14]. The latter configuration could have appeal due to its relatively smaller input power and smaller pulse heating on cavity walls, as compared to the others; furthermore, its longitudinal field can have a spatially tapered distribution which is favorable from the beam dynamics standpoint. But the price to be paid for these virtues is a reduction of the frequency up-shift factor from $4 \gamma^{2}$ to $2 \gamma^{2}$.

Analysis of these three cases begins with the equations of particle motion, here expressed in a Cartesian system of coordinates with $z$ along the principal direction of particle motion. It follows from Eq. (5) that, in order to produce radiation of $\mathrm{nm}$ wavelength using $1 \mathrm{~cm}$ wavelength radiation to excite the cavities, one needs an electron beam with energy of about $1 \mathrm{GeV}\left(\gamma_{0} \sim 2 \times 10^{3}\right)$. We consider interactions of an ultrarelativistic particle in plane wavelike rf fields (with $E_{z}=E_{y}=0$ ) to be weak enough so that the Lorentz energy factor $\gamma$ can be represented as

$$
\gamma(t)=\gamma_{0}+\Delta \gamma(t)
$$

where $\Delta \gamma(t)$ satisfies

$$
\frac{\Delta \gamma(t)}{\gamma_{0}} \ll 1
$$

In this case we can write the equation for transverse particle motion,

$$
\frac{d\left(m c \gamma \beta_{x}\right)}{d t}=e\left(E_{x}-\beta_{z} H_{y}\right),
$$

in the lowest approximation of perturbation theory, neglecting changes of the particle energy caused by the transverse electric field, as

$$
\frac{d \beta_{x}}{d t}=\frac{e}{m c \gamma_{0}}\left(E_{x}-H_{y}\right)
$$

The equation for particle energy is

$$
\frac{d \gamma}{d t}=\frac{e}{m c} \beta_{x} E_{x}
$$

\section{A. Traveling-wave (TW) undulator}

In a traveling-wave (TW) undulator [9], only the counterpropagating wave with constant amplitude (either TE or TM type) is assumed to be present within the interval $z=[0, L]$. In the TE-mode case, we represent the rf fields 
of the counterpropagating wave relative to a particle moving in the $+z$ direction as

$$
\begin{aligned}
& E_{x}=E_{\text {count }}=H_{0} \frac{k}{h} \cos (\omega t+h z+\varphi), \\
& H_{y}=H_{\text {count }}=-H_{0} \cos (\omega t+h z+\varphi),
\end{aligned}
$$

where $\varphi$ is the phase of the wave relative to the particle. Note that when considering the case of a cavity near to cutoff where $h \rightarrow 0$, then the wave magnetic field $H$ is negligibly small in comparison with the electric field $E$. In the TM-mode case, the fields can be written in a similar form, namely,

$$
\begin{aligned}
E_{x} & =E_{\text {count }}=H_{0} \frac{h}{k} \cos (\omega t+h z+\varphi), \\
H_{y} & =H_{\text {count }}=-H_{0} \cos (\omega t+h z+\varphi),
\end{aligned}
$$

where if $h=0$, then $E_{x}=0$, but $H_{y} \neq 0$. Substituting Eqs. (16) and (17) into Eq. (14), we obtain two similar equations, namely,

$$
\frac{d \beta_{x}}{d \vartheta}=-\frac{K_{\mathrm{TE}}}{\gamma_{0}} \cos (\vartheta+\varphi)
$$

in the TE case and

$$
\frac{d \beta_{x}}{d \vartheta}=-\frac{K_{\mathrm{TM}}}{\gamma_{0}} \cos (\vartheta+\varphi)
$$

in the TM case, where we have replaced laboratory $z$ and $t$ variables by the normalized time variable $\vartheta$, with $t=$ $\vartheta / k_{u} c$, where $k_{u}$ was defined in Eq. (1). The amplitude coefficients $K_{\mathrm{TE}}$ and $K_{\mathrm{TM}}$ are given by

$$
K_{\mathrm{TE}}=\frac{e H_{0} \lambda_{g}}{2 \pi m c^{2}},
$$

and

$$
K_{\mathrm{TM}}=\frac{e H_{0} \lambda}{2 \pi m c^{2}} .
$$

In equations that follow, subscripts are omitted; so that $K=K_{\mathrm{TE}}$ in the TE case or $K=K_{\mathrm{TM}}$ in the TM case.

The solutions for Eqs. (18) and (19) are

$$
\beta_{x}=-\frac{K}{\gamma_{0}} \sin (\vartheta+\varphi)+\beta_{x 0},
$$

where the constant velocity $\beta_{x 0}$ depends on initial conditions. If $\beta_{x}(0)=0$ then

$$
\beta_{x 0}=\frac{K}{\gamma_{0}} \sin \varphi
$$

In order to find the dependence of transverse particle coordinate $x$ upon $\vartheta$, Eq. (22) is integrated, to yield

$$
X(\vartheta)=\frac{K}{\gamma_{0}} \cos (\vartheta+\varphi)+\beta_{x 0} \vartheta-\frac{K}{\gamma_{0}} \cos \varphi,
$$

where the dimensionless variable $X=x k_{u}$ is introduced, and the paraxial condition for the reference beam $X(0)=0$ is assumed.

In order to obtain undulator radiation with wavelength $\lambda_{s} \sim 1 \mathrm{~nm}$ using $\sim 1 \mathrm{~cm}$ wavelength $\mathrm{rf}$ cavity fields, a high energy beam is necessary $\left(\gamma_{0} \sim 2 \times 10^{3}\right)$ in accordance with Eq. (5). Taking into account that a realistic undulator parameter could be as high as $K \sim 1$, one concludes that transverse velocity is much less than the light velocity, that is

$$
\frac{K}{\gamma_{0}} \ll 1 .
$$

This inequality justifies a posteriori the assumption made in Eqs. (11)-(15).

Note that the constant transverse velocity $\beta_{x 0}$ appearing in Eqs. (22)-(24) should be small enough in order that transverse displacements at the end of the undulator of length $L$ are less than the radius of the electron beam, i.e.,

$$
\left|\beta_{x 0}\right| L \leq r_{b} .
$$

This requirement agrees with that in Ref. [2], where it was proven that the highest gain and minimal diffraction losses are experienced for an optical Gaussian mode with wave-beam waist approximately equal to the electron beam radius. The inequality Eq. (26) determines acceptable values of the injection phase for a given beam radius. The smallest possible wave-beam waist and electron beam radius corresponding to the highest gain are determined by the condition that the Fresnel parameter $N_{F}$ is near unity for propagation of the optical wave beam through the undulator, namely,

$$
N_{F} \equiv \frac{r_{b \min }^{2}}{\lambda_{s} L} \sim 1
$$

In the paraxial wave approach $\left(\lambda_{s}=\lambda / 4 \gamma_{0}^{2}\right)$ the acceptable range of the injection phases is written substituting Eq. (23) in Eq. (26) with Eq. (27) taken into account,

$$
\Delta \varphi \leq 2 \arcsin \left(\frac{1}{2 K} \sqrt{\frac{\lambda}{L}}\right)
$$

According to Eq. (28) in the $1 \mathrm{~m}$ long rf undulator, operating at wavelength $1 \mathrm{~cm}$ with $K=0.4$, electron bunches should be injected in the phase interval $\pm 7^{\circ}$ which in a paraxial approach corresponds to the longest acceptable bunch length $\sim 0.4 \mathrm{~mm}$.

Let us now determine the velocity and transverse coordinates which a particle would have at the exit plane of the undulator. This is especially important if an undulator consists of several sections in tandem. In such an undulator the ideal condition is that particles which finish their flight in a given section have zero transverse velocities and transverse shifts (much less than the beam radius). From Eqs. (23)-(25), this requirement is satisfied for particles 
near the injection angles $\varphi=0$ or $\pi$, supplemented by the condition that the particle makes an integer number of oscillations within the undulator section, namely,

$$
\vartheta_{\text {end }} \equiv L K_{u}=2 \pi n,
$$

where $n$ is an integer.

Note that Eq. (29) implies generally that the undulator length, frequency, and waveguide propagation constant cannot be chosen arbitrarily. Naturally these parameters are already not independent, since they must conform to the cavity eigenmode.

The disadvantage of the TW undulator to operate with short bunches (comparing to rf wavelength) and to have fixed length [constrained by Eq. (29)] arise from the abrupt jumps in rf field amplitudes when particles enter and leave the cavity. In undulators employing static magnetic fields this problem is circumvented by the use of smooth field tapers at each end of the undulator [16]. By analogy, field tapers are applicable for rf undulators as well.

Let us consider the equations of motion in an undulator with nonuniform $K$ parameter,

$$
\frac{d \beta_{x}}{d \vartheta}=-\frac{K(\vartheta)}{\gamma_{0}} \cos (\vartheta+\varphi),
$$

and analyze motion in a linearly increasing field at $0 \leq$ $z \leq l_{\text {tap }}$ and in a constant field at $z>l_{\text {tap }}$, where $l_{\text {tap }}$ is a length of the taper,

$$
\begin{aligned}
& K(\vartheta)=K_{0} \frac{\vartheta}{\vartheta_{\text {tap }}}, \quad 0 \leq \vartheta \leq \vartheta_{\text {tap }}, \\
& K(\vartheta)=K_{0}, \quad \vartheta>\vartheta_{\text {tap }},
\end{aligned}
$$

where $\vartheta_{\text {tap }}=l_{\text {tap }} k_{u}$. When the length of the taper is big enough, i.e.,

$$
\vartheta_{\text {tap }}=l_{\text {tap }} k_{u} \gg 1,
$$

the solution of Eqs. (30) and (31) is given neglecting terms $1 / \vartheta_{\text {tap }}^{2}$,

$$
\begin{aligned}
\beta_{x}= & -\frac{K(\vartheta)}{\gamma_{0}} \sin (\vartheta+\varphi) \\
& -\frac{1}{\vartheta_{\text {tap }}} \frac{K_{0}}{\gamma_{0}}[\cos (\vartheta+\varphi)-\cos \varphi], \quad 0 \leq \vartheta \leq \vartheta_{\text {tap }} \\
\beta_{x}= & -\frac{K_{0}}{\gamma_{0}} \sin (\vartheta+\varphi)+\beta_{x 0}, \quad \vartheta>\vartheta_{\text {tap }}
\end{aligned}
$$

where

$$
\beta_{x 0}=-\frac{1}{\vartheta_{\text {tap }}} \frac{K_{0}}{\gamma_{0}}\left[\cos \left(\vartheta_{\text {tap }}+\varphi\right)-\cos \varphi\right] .
$$

The solution given by Eqs. (33) and (34) represents for arbitrary electron injection phase $\varphi$ smoothly growing oscillations in the taper section and sinelike oscillations of the constant amplitude behind this taper. Note that constant lateral velocity [Eq. (34)] is zero, if $\vartheta_{\text {tap }}=2 \pi m$, where $m$ is an integer $(m>0)$. In the general case $\beta_{x 0}$ is not zero, but small for arbitrary injection phase and taper length [Eq. (32) is assumed to be valid],

$$
\left|\beta_{x 0}\right| \leq \frac{1}{\vartheta_{\text {tap }}} \frac{2 K_{0}}{\gamma_{0}} \ll \frac{K_{0}}{\gamma_{0}}
$$

and, therefore, the inequality Eq. (26) can be satisfied even for a full range of the injection phases.

We now consider (in the context of perturbation theory) the variations of particle energy due to work done by the transverse electric field. Equation (15) leads to the solution, using Eqs. (16), (17), (20), and (21), expressed in dimensionless variables, as

$$
\begin{aligned}
\gamma(\vartheta) & \equiv \gamma_{0}+\Delta \gamma(\vartheta) \\
& =\gamma_{0}-v \cos (2 \vartheta+2 \varphi)+v \cos 2 \varphi
\end{aligned}
$$

where

$$
\vartheta=\frac{K^{2} k}{\gamma_{0}(k+h)} .
$$

So $\Delta \gamma / \gamma_{0} \sim K^{2} / \gamma_{0}^{2}$. Let us also employ the relationship for $\gamma_{z}$ in terms of the full energy factor $\gamma$, namely,

$$
\gamma_{z}^{2}(\vartheta)=\frac{\gamma^{2}(\vartheta)}{1+\gamma^{2}(\vartheta) \beta_{x}^{2}(\vartheta)} .
$$

Substituting Eqs. (22), (23), and (36) for $\beta_{x}$ and $\gamma$ in an untapered undulator, and averaging on oscillations of $\beta_{x}^{2}$ and $\gamma^{2}$, one obtains

$$
\gamma_{z 0}^{2}=\frac{\gamma_{0}^{2}}{1+K^{2} / 2+K^{2} \sin ^{2} \varphi}+2 v \gamma_{0} \cos 2 \varphi+o\left(\frac{1}{\gamma_{0}}\right) .
$$

The second term in Eq. (39), being proportional to $K^{2}$, is $\gamma_{0}^{2}$ times smaller than the first term. If one inserts Eq. (39) into Eq. (2), and neglects all terms in Eq. (39) excepting the first, the result in general differs from Eq. (5) due to a constant lateral velocity, namely,

$$
\lambda_{s}=\lambda_{s} \frac{1+K^{2} / 2+K^{2} \sin ^{2} \varphi}{2 \gamma_{0}^{2}}
$$

For $\varphi=0$ (i.e., for a particle is injected at the field maximum), Eq. (40) reverts to Eq. (5). The difference can be understood since the constant lateral velocity Eq. (23) on top of the oscillatory velocity leads to a small reduction of the longitudinal particle energy, and as a consequence the output wavelength $\lambda_{s}$ is longer.

The constraint on injection phase in accordance with Eqs. (26)-(28) and as exhibited in Eq. (40) has several important consequences. First, the electron beam must have a big enough radius so that transverse displacements do not cause particles to wander out of the optical beam in order to keep high gain; and second, particles injected at different phases will produce output radiation of different 
wavelengths. In order to avoid such incoherence, short enough bunches as compared with rf wavelength or a tapered undulator should be used. In the tapered undulator there are not such strict conditions for bunch length, as given by Eqs. (26)-(28), because particle off-axis shift is small [Eq. (35)]. If the taper length is as high as Eq. (32) requires, but it is still much less than the total length of the undulator, the optical spectrum of the rf undulator is determined by a homogeneous undulator part. The resonant light frequency of each electron is given by Eq. (5), where one should use the $K$ parameter for the homogeneous part of the undulator.

\section{B. Standing wave (SW) undulator}

High field amplitude to wiggle particles is reachable in high- $Q$ resonators where the field structure is usually a standing wave. In a standing wave (SW) rf undulator the operating mode consists of a copropagating wave and a counterpropagating wave. In 1987, Tran, Danly, and Wurtele [14] examined the standing wave resonator concept. They studied plane waves, which have constant amplitude across the plane transverse to the particle motion and have only transverse fields. Their analysis found that, for $K \sim 1$, the radiation spectrum splits into many separate modes because the motion of the electrons contains many harmonics. For while the counterpropagating wave causes the desired FEL quiver electron motion with a relativistic Doppler up-shift of Compton scattered photons, the copropagating wave also interacts with the electrons and produces dominant low-frequency motions.

To further examine the standing wave resonator concept and suppress the radiation spectrum degradation due to the copropagating wave, let us start studies of a standing wave undulator with the simplest case, where the field consists of copropagating and counterpropagating waves of the same type, namely,

$$
\begin{aligned}
E_{x}= & E_{\mathrm{co}}+E_{\text {count }}=H_{0} \frac{k}{h} \cos \left(-\omega t+h z+\psi_{E}\right) \\
& +H_{0} \frac{k}{h} \cos (\omega t+h z+\varphi), \\
H_{y}= & H_{\mathrm{co}}+H_{\mathrm{count}} \\
= & H_{0} \cos \left(-\omega t+h z+\psi_{E}\right)-H_{0} \cos (\omega t+h z+\varphi),
\end{aligned}
$$

in the TE-mode case, and

$$
\begin{aligned}
E_{x} & =E_{\mathrm{co}}+E_{\mathrm{count}} \\
& =H_{0} \frac{h}{k} \cos \left(-\omega t+h z+\psi_{H}\right)+H_{0} \frac{h}{k} \cos (\omega t+h z+\varphi), \\
H_{y} & =H_{\mathrm{co}}+H_{\mathrm{count}} \\
& =H_{0} \cos \left(-\omega t+h z+\psi_{H}\right)-H_{0} \cos (\omega t+h z+\varphi),
\end{aligned}
$$

in TM-mode case. Substituting Eqs. (41) and (42) in Eq. (14), we obtain the equation

$$
\frac{d \beta_{x}}{d \vartheta}=-\frac{K}{\gamma_{0}}[\delta \cos (\vartheta \delta+\psi)+\cos (\vartheta+\psi)],
$$

where we used a designation for cutoff factor $\delta=$ $(k-h) /(k+h)$ which can range from 0 (for paraxial wave) to 1 (for near to cutoff wave). This factor provides a measure of the relative difference between guide and free-space rf wavelengths. $\psi=\psi_{E}$ or $\psi=\psi_{H}$ are phases of the copropagating waves relative to phases of the corresponded counterpropagating waves in TE and TM cases, respectively. Here again we take $K=K_{\mathrm{TE}}$ in the TE case or $K=K_{\mathrm{TM}}$ in the TM case. The solution of Eq. (43) is

$$
\beta_{x}=-\frac{K}{\gamma_{0}}[\sin (\vartheta \delta+\psi)+\sin (\vartheta+\varphi)]+\beta_{x 0},
$$

and

$$
X=\frac{K}{\gamma_{0}}\left[\frac{1}{\delta} \cos (\vartheta \delta+\psi)+\cos (\vartheta+\varphi)\right]+\beta_{x 0} \vartheta+X_{0}
$$

where

$$
\begin{aligned}
\beta_{x 0} & =\frac{K}{\gamma_{0}}[\sin \psi+\sin \varphi], \\
X_{0} & =-\frac{K}{\gamma_{0}}\left[\frac{1}{\delta} \cos \psi+\cos \varphi\right] .
\end{aligned}
$$

The first terms in the square brackets of Eqs. (44) and (45) describe electron motion in the field of the copropagating wave, while the second terms are from the counterpropagating wave. The first terms cannot be neglected in comparison with second terms for any $K$ value and/or any injection phase $\psi$ of electrons relative to the copropagating wave. This circumstance, apparently overlooked in the first publications devoted to rf undulators (e.g. [6]), leads as it will be shown to crucial spectrum degradation in the case of large $K$ values.

Here let us assume that $\delta$ is small, i.e. $k \approx h$ according to the paraxial approach. This condition causes the amplitude of the first term in the brackets of Eq. (45) for the transverse displacement to always exceed the second term. This automatically means that in this case only electron beams of big enough radius can be used, i.e.,

$$
\frac{K}{\gamma_{0} k_{u} \delta}<r_{b}
$$

for otherwise extreme particle excursions will seriously degrade the SASE radiation pattern, will reduce gain in the case of undulator based FELs, and might cause problems with beam optics in rf undulators consisting of several sequential sections. A high undulator parameter and transverse coherence of the output optical radiation appear incompatible. The opposite case, when $\delta$ is close to unity, 
is considered in the next section regarding the near to cutoff undulator cavity.

If a particle performs many oscillations in the field of the copropagating wave so that

$$
L k_{u} \delta \gg 2 \pi,
$$

one might formally use Eqs. (44) and (46) in order to insert them into Eq. (38), and to obtain by averaging

$$
\lambda_{s}=\lambda_{u} \frac{1+K^{2}+\beta_{x 0}^{2} \gamma_{0}^{2}}{2 \gamma_{0}^{2}} .
$$

In the numerator the 2 times higher term in $K^{2}$, as compared with Eq. (5), might leave an erroneous impression that due to the copropagating wave the effective $K$ parameter becomes bigger. In fact, only the shift in frequency is increased, but the beam microbunching that depends on the deflecting force (i.e. on $K$ ) is not larger. Moreover, as it was mentioned, deflections of electrons far from the optical beam lead to reduction of gain and microbunching effect.

Note that Eq. (49) gives the frequency of the main resonance with the counterpropagating wave, while large magnitude electron motion in the copropagating wave produces additional harmonics in the spectrum.

The term $\beta_{x 0}$ in Eqs. (44) and (46) might be zero, if proper boundary conditions for fields at $\vartheta=0$ and at $\vartheta=L k_{u}$ are imposed. For example, if the condition corresponding to reflection by a perfect conducting wall

$$
\psi=\varphi+\pi
$$

is imposed, then the constant transverse velocity given by Eq. (46) is exactly zero. At the end of the undulator the transverse velocity and transverse coordinate might also be zero, if two conditions are satisfied simultaneously, that is, if

$$
\vartheta_{\text {end }} \equiv L k_{u}=2 \pi n_{1}, \quad \vartheta_{\text {end }} \delta \equiv L k_{u} \delta=2 \pi n_{2},
$$

where $n_{1}$ and $n_{2}$ are integers. To satisfy these requirements together with that necessity to satisfy resonance at a given frequency seems difficult.

Accurate beam dynamics studies have been carried out using parameters close to those for an undulator for generating $1 \mathrm{~nm}$ radiation from a beam with incident energy of $660 \mathrm{MeV}$ at an rf frequency $f=34 \mathrm{GHz}$. The length of the $\mathrm{TE}_{11}$ mode undulator is taken as $50 \mathrm{~cm}$, and the injection phase is taken as $\varphi=0$. The condition given in Eq. (49) was used to avoid particle trajectories deviating far from the injection axis at $x=y=0$. The conditions of Eq. (51) could not be satisfied strictly. In order to calculate particle trajectories and individual radiation spectra of electrons we used integration of the exact system of differential equations. (See Appendix B for details.) Results are represented in Figs. 2 and 3. All trajectories in Fig. 2 show small-scale quiver motion (due to the counterpropagating wave) on a background of large-scale oscillations (due to the

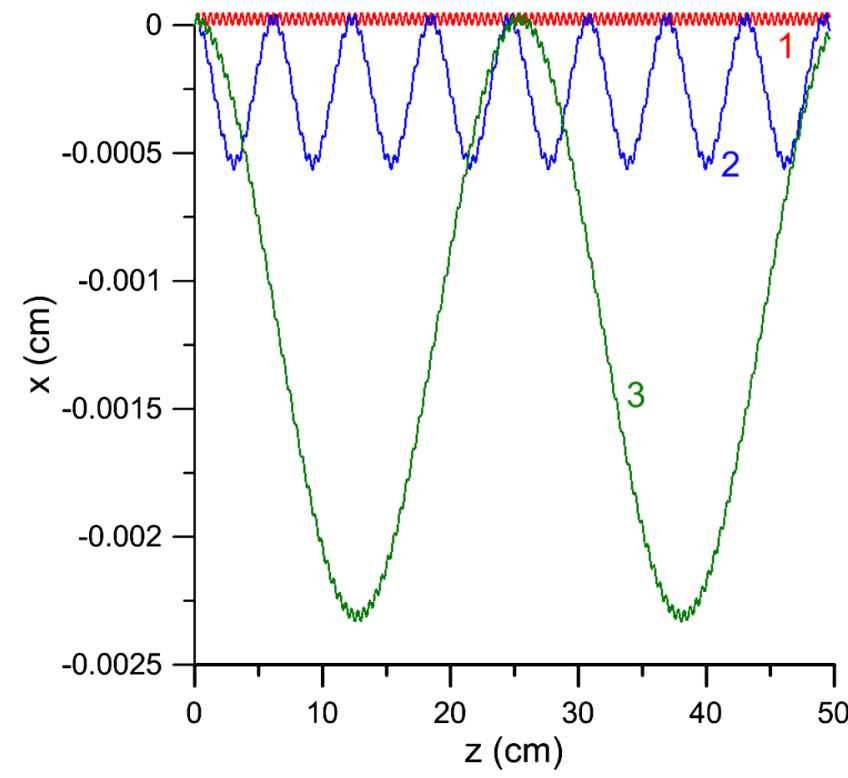

FIG. 2. Motion of sample electrons with $K=0.4$. 1 -in the field of counterpropagating wave only $(R=4.9 \mathrm{~mm}, \delta=$ $\left.8.3 \times 10^{-2}\right) ; 2$-in the field of copropagating and counterpropagating waves together $\left(R=4.9 \mathrm{~mm}, \delta=8.3 \times 10^{-2}\right)$; and 3 -in the field of copropagating and counterpropagating waves together $\left(R=9.6 \mathrm{~mm}, \delta=1.9 \times 10^{-2}\right)$.

copropagating wave). In order to prove that these largescale oscillations are caused by the copropagating wave we also investigated particle motion using only the counterpropagating wave of the same waveguide where beam motion in the standing wave was calculated (curves 1 in Figs. 2 and 3). The magnitude of the large-scale oscillations and their period increase as the difference shrinks between the guide and vacuum wavelengths. This unwelcome trajectory distortion arises because the forward wave (or copropagating wave) is in near synchronism with electrons during their transit along the waveguide. The condition of this unwanted synchronism depends on the difference between the electron velocity $c$ and the phase velocity $v_{\text {ph }}$ of the copropagating wave, i.e.,

$$
\left(v_{\mathrm{ph}}-c\right) L / c \leq \lambda / 2 .
$$

Figure 4 shows also that if a particle makes several oscillations in the copropagating wave along the undulator, the trajectories have large transverse deflections, and the radiation spectra of this particle (Fig. 3) exhibit many lowamplitude maxima even in the case when the final particle distortion at the end of the undulator $(z=L)$ is small. The frequencies produced in the standing wave differ from frequencies produced by the same particles in the counterpropagating wave alone (Fig. 3). These observations are valid even for a small but finite value of the $K$ parameter.

Let us examine also the case which is opposite to the condition given by Eq. (48), namely,

$$
L k_{u} \delta \ll 2 \pi
$$




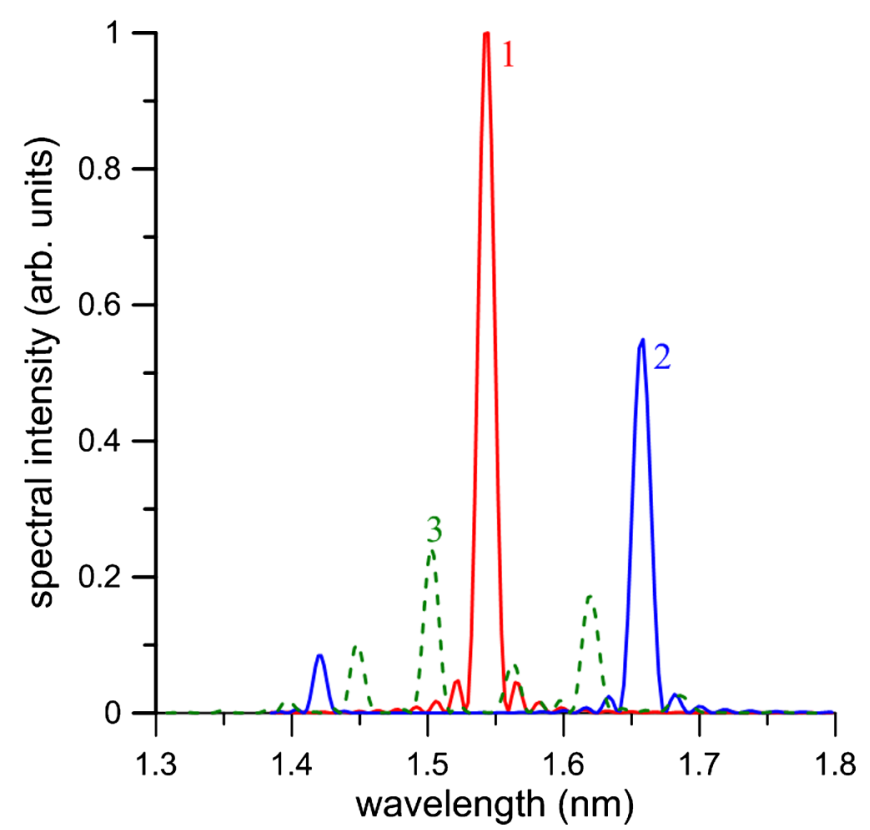

FIG. 3. Radiated spectra of sample electrons $(K=0.4)$ : 1 -spectrum produced by a counterpropagating wave only $\left(R=4.9 \mathrm{~mm}, \quad \delta=8.3 \times 10^{-2}\right) ; 2$-spectrum produced by copropagating and counterpropagating waves together $\left(R=4.9 \mathrm{~mm}, \quad \delta=8.3 \times 10^{-2}\right)$; and 3 -spectrum produced by copropagating and counterpropagating waves together $\left(R=9.6 \mathrm{~mm}, \delta=1.9 \times 10^{-2}\right)$.

which states that particles do not experience much slip in phase relative to the copropagating wave. Note this allows the second condition in Eq. (51) to be satisfied when $n_{2} \approx 0$. The boundary condition Eq. (50) needs to be satisfied so that steady transverse deflection is absent. In this case the asymptotic solution to Eq. (45) is

$$
X=-\frac{K}{\gamma_{0}} \sin \vartheta
$$

This solution describes harmonic wiggling which is possible if a particle is injected near a node of the copropagating wave (optimal phase $\varphi_{\mathrm{opt}}= \pm \pi / 2$ ). This favorable condition can be maintained until the particle slips in relative phase. Typical particle trajectories and corresponded radiation spectra for this case $(R=29 \mathrm{~mm}$, i.e. $\delta=2 \times 10^{-3}$ ) are plotted in Fig. 4 for three values of the difference between injection phase and the optimal value using exact 3D equations of motion. Figure 4(b) shows that radiation spectra are distorted even for small variations of the injection phase. This approach to obtain a clean radiation spectrum demands a waveguide of large diameter, which in turn requires large rf power levels.

Let us also analyze possible profits of tapers in SW undulator, because one can expect that similar to TW undulator, the use of the taper sections will alleviate the constraints imposed by Eqs. (50) and (51). Trajectories and spectra of the test particles in $\mathrm{SW}$ undulator with $8 \mathrm{~cm}$ long

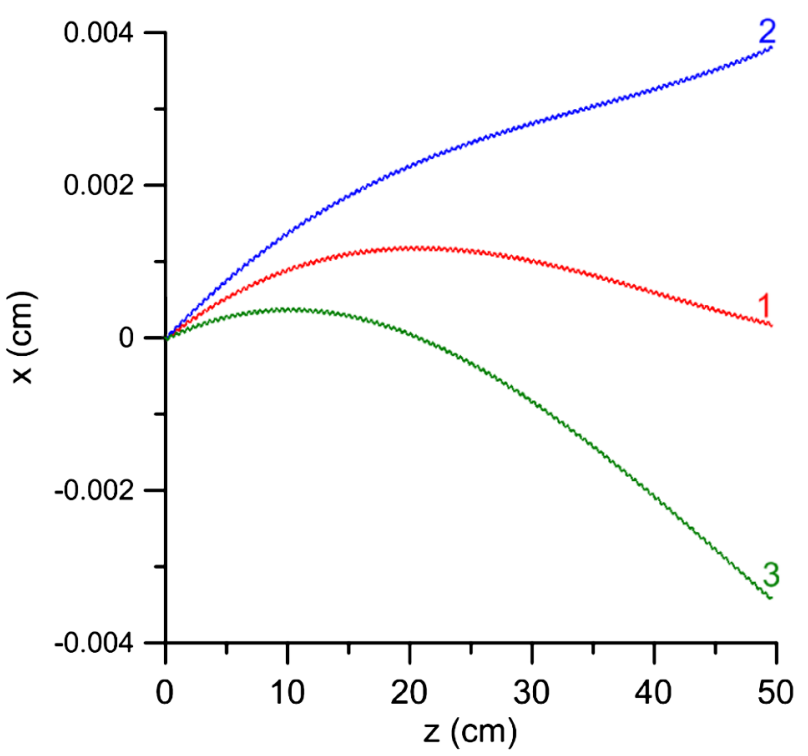

(a)

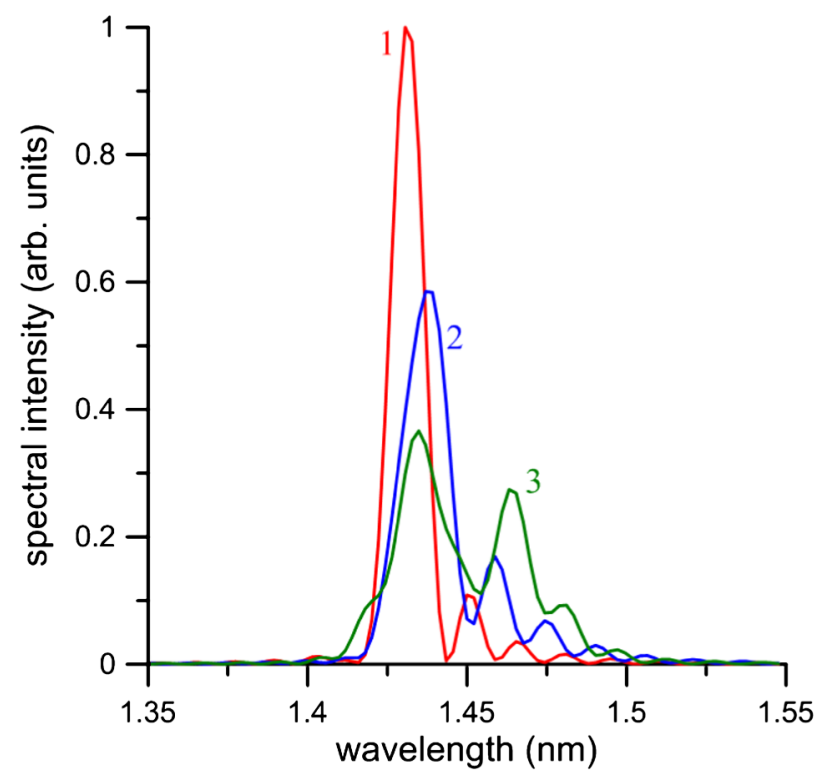

(b)

FIG. 4. Motion of test electrons with different injection phases with respect to the optimal phase $\varphi_{\text {opt }}$, with $K=0.4$ : (a) trajectories of particles; (b) radiated spectra. (1) $\Delta \varphi=0^{\circ}$; (2) $\Delta \varphi=+10^{\circ}$; and (3) $\Delta \varphi=-10^{\circ}$.

linear tapers are shown in Figs. 5(a) and 5(b) respectively for three values of the injection phases where all simulation parameters correspond to that used in Figs. 2 and 3 for waveguide radius $4.9 \mathrm{~mm}$. The curve 1 in Fig. 5(a) corresponds to the optimized phase which provides close to zero off-axis shift in the end of the undulator. Two other curves in this figure, plotted for relative injection phases $-\pi / 4$ and $+\pi / 4$, confirm that the final lateral shift in these cases is not much dependent on injection phase. Nevertheless, large-scale deviations of the particles, caused by the copropagating wave, remain. A quality of the spectra 


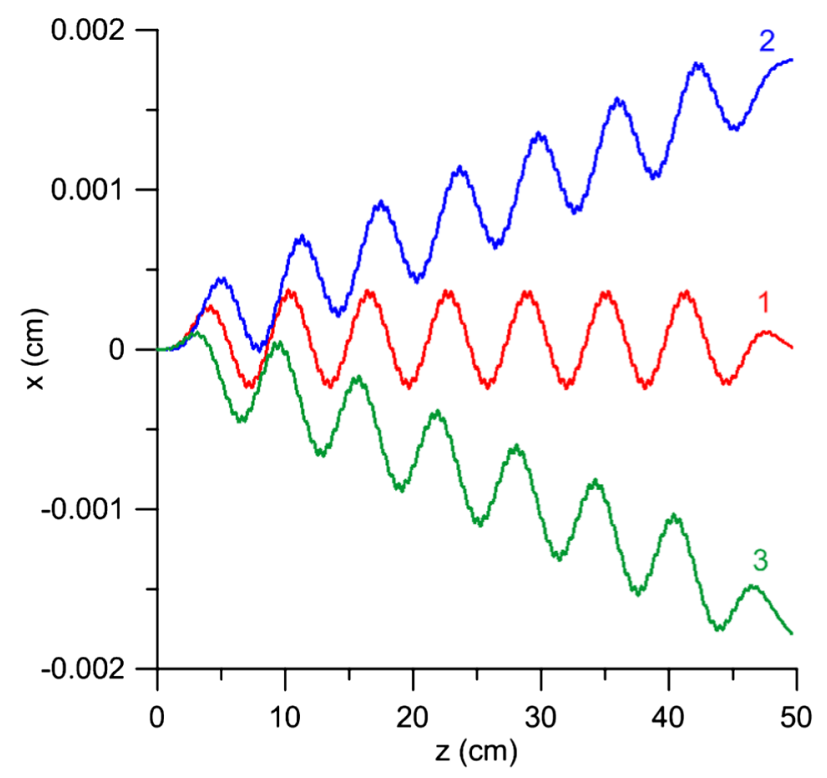

(a)

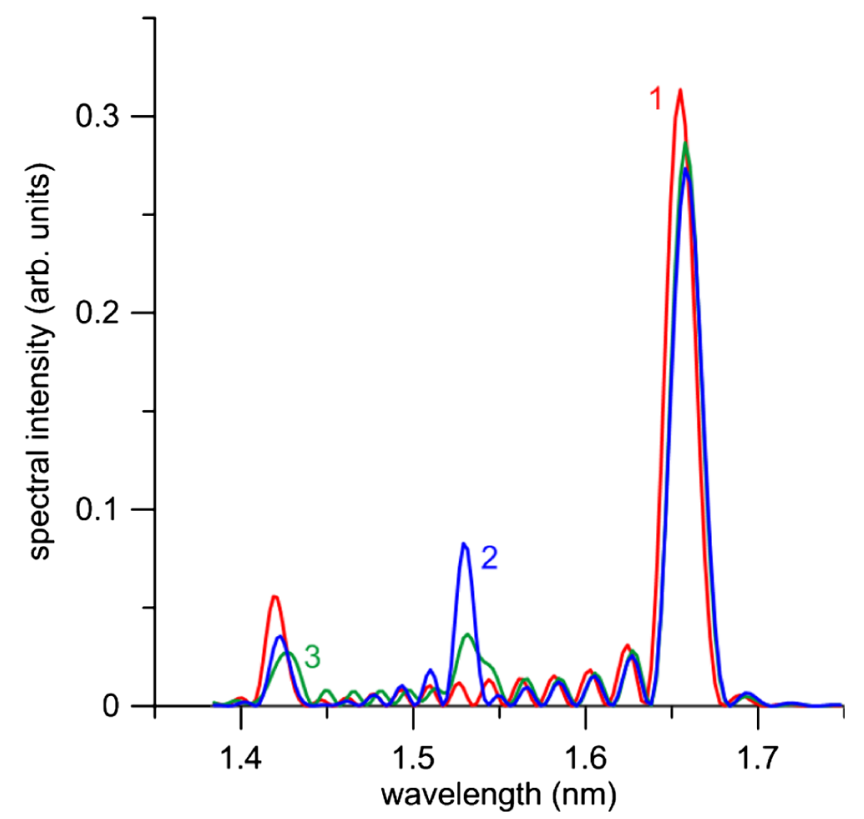

(b)

FIG. 5. Motion of test electrons in a tapered SW undulator with different injection phases with respect to the optimized phase $(K=0.4)$ : (a) trajectories of particles; (b) radiated spectra. (1) $\Delta \varphi=0^{\circ}$; (2) $\Delta \varphi=-45^{\circ}$; and (3) $\Delta \varphi=+45^{\circ}$.

[Fig. 5(b)] in the tapered undulator is not improved in comparison with spectrum quality in Fig. 3 (curve 2) for the untapered case. Note that data in Fig. 5(a) are normalized with the same coefficient like data in Fig. 3.

Two ways can be used to avoid beam distortions caused by the copropagating waves either in the tapered or untapered undulator. The first approach is to use a cavity where the copropagating and counterpropagating waves are modes with different transverse field structures, so that electrons interact only with the counterpropagating wave, provided the copropagating mode has zero fields along the beam trajectory $[17,18]$.

The second approach is to use a near to cutoff resonator where copropagating and counterpropagating waves are similar to one another as shown in Eqs. (44) and (45) with $\delta \rightarrow 1[17,18]$. This approach is discussed in the next section.

A possible arrangement to realize the first of these schemes would employ an on off-axis beam which interacts in a cavity structure, where the $\mathrm{TE}_{01}$ mode counterpropagates and the $\mathrm{TE}_{02}$ mode copropagates. This way allows a standing wave undulator to resemble a travelingwave undulator, by minimizing interactions with the copropagating wave, in which case the equations obtained in the previous section for a TW undulator become valid.

A possible advantage of an undulator where the electron beam is placed in the region of zero fields of the copropagating wave is that the ponderomotive (Miller) force [12] from the copropagating wave can provide beam focusing. It is natural to place the beam at the maximum of the counterpropagating wave field. The ponderomotive force of the counterpropagating wave is zero in that point. But if the orbit is slightly deflected from this equilibrium position, the particle sees a force which deflects it away from the axis. For the TE-mode case, the defocusing force due to the counterpropagating wave is given by

$$
F_{\text {count }}=-\left(\frac{e\left(1-\beta_{z 0} h / k\right)}{2 m \omega\left(1+\beta_{z 0} h / k\right)}\right)^{2} \gamma_{z 0} \nabla_{\perp}\left|E_{\text {count }}\left(r_{\perp}\right)\right|^{2} \geq 0,
$$

where the electric field is taken in the laboratory system of the coordinates. Transverse fields of the copropagating wave may be zero at the beam position and rise away from the beam, so it can provide focusing. Note that focusing of the copropagating wave is potentially much stronger than the defocusing of the counterpropagating wave (except for the near to cut off case where $h \rightarrow 0$ ), namely,

$$
F_{\mathrm{co}}=-\left(\frac{e\left(1+\beta_{z 0} h / k\right)}{2 m \omega\left(1-\beta_{z 0} h / k\right)}\right)^{2} \gamma_{z 0} \nabla_{\perp}\left|E_{\mathrm{co}}\left(r_{\perp}\right)\right|^{2} \leq 0,
$$

due to the much smaller denominator.

\section{Near to cutoff (NC) undulator}

A near to cutoff undulator is a special case of the standing wave undulator. Nevertheless, it is discussed separately because of its unusual features. Optical radiation frequencies of both the copropagating and the counterpropagating waves tend to be equal. Such mode superposition could be excited in a so-called barrel-like cavity, i.e., in a section of uniform waveguide with narrowing regions at each end (the detailed design will be discussed in the future paper). In such cavity of length $L$ both 
waves have nearly zero propagation constants $h_{\text {co }}$ and $h_{\text {count }}$, namely,

$$
h_{\text {co }} \equiv-h_{\text {count }}=\frac{\pi}{L} \text {. }
$$

Taking into account that $\delta$ is very close to unity in a long (comparing to a wavelength) cavity and $\psi=\varphi$, Eq. (43) takes the asymptotic form with both waves contributing to the relevant swing field,

$$
\frac{d \beta_{x}}{d \vartheta}=-\frac{2 K(\vartheta)}{\gamma_{0}} \cos (\vartheta+\varphi) .
$$

The field distribution in a near to cutoff cavity of the length $L$ is close to be sinusoidal, i.e.,

$$
2 K(\vartheta)=K_{0} \sin \left(\frac{\pi \vartheta}{\vartheta_{\text {end }}}\right),
$$

where $\vartheta_{\text {end }}=L k_{u}$ is assumed to be much more than $\pi$. The solution of Eq. (58) with field distribution given by Eq. (59) describes particle's oscillations which exactly follow for the field shape, i.e.,

$$
\begin{aligned}
\beta_{x}= & -\frac{K_{0}}{\gamma_{0}} \sin \left(\frac{\pi \vartheta}{\vartheta_{\text {end }}}\right) \sin (\vartheta+\varphi)-\frac{K_{0}}{\gamma_{0}} \frac{\pi}{\vartheta_{\text {end }}} \cos \left(\frac{\pi \vartheta}{\vartheta_{\text {end }}}\right) \\
& \times \cos (\vartheta+\varphi)+\beta_{x 0}+o\left(\frac{\pi^{2}}{\vartheta_{\text {end }}^{2}}\right) .
\end{aligned}
$$

The second and the constant terms in Eq. (60) are small under the assumptions taken,

$$
\left|\beta_{x 0}\right|=\frac{\pi}{\vartheta_{\text {end }}} \frac{K_{0}}{\gamma_{0}}|\cos \varphi| \ll \frac{K_{0}}{\gamma_{0}} .
$$

Therefore, all particles injected near the axis produce (independently of injection phase) spectra whose maxima correspond the same wavelength,

$$
\lambda_{s}=\lambda \frac{1+K_{0}^{2} / 2+K_{0}^{2}\left(\frac{\pi}{\vartheta_{\text {end }}}\right)^{2} \cos ^{2} \varphi}{2 \gamma_{0}^{2}},
$$

because the third term in the numerator of Eq. (62) is small compared to the other two terms In such an undulator a long bunch in comparison with rf wavelength is an appealing possibility (as in the tapered TW undulator). The penalty of using this structure is a reduction by a factor of 2 in the up-shift in optical radiation frequency, in comparison with the far-from-cutoff case, due to the nearly zero propagation constant of the scattering rf wave. Methods to excite this type of cavity, spectral characteristics of optical radiation generated in this undulator, and influence of geometry errors on radiation quality will be described in a forthcoming publication.

\section{CONCLUSION}

An rf undulator fed by a high-power cm- or mmwavelength $\mathrm{rf}$ source is an appealing device for exciting a $\mathrm{GeV}$ electron beam to produce radiation in the $\mathrm{nm}$ - wavelength regime. Possible rf undulator configurations can be classified as a traveling-wave undulator, a standing wave undulator, and a near to cutoff undulator. In comparison with a conventional DC-magnet undulator, rf undulators employing a standing wave cavity have features discussed for the first time in this paper, caused by an existence of the copropagating wave. The copropagating wave might cause problems for beam optics (due to largescale off-axis deviation of particles), for providing high power gain, and for quality of a generated radiation. Several possible solutions are introduced to choose the best undulator configuration, such as using a dual mode standing wave undulator or a near to cutoff undulator to overcome the trajectory distortion due to the copropagating wave, implementing field taper section in a traveling-wave undulator or using near to cutoff undulator to broaden electron beam injection acceptance.

Detailed engineering considerations and the design realization of each configuration is planned to be published in the future.

\section{ACKNOWLEDGMENTS}

This research was supported in part by the U.S. Department of Energy, Office of High Energy Physics.

\section{APPENDIX A: $K$ PARAMETER FOR AN RF UNDULATOR}

Let us assume that the undulator $K$ parameter is small enough so that the transverse wiggling velocity is much less than the longitudinal velocity of beam electrons, in accordance with comments accompanying Eq. (25). In this case the modulus of total momentum of a moving particle is approximately constant along the particle trajectory, i.e.,

$$
|\mathbf{p}|=m c \gamma_{0}=\text { const. }
$$

The equation of motion is

$$
\frac{d \mathbf{p}}{d t}=\mathbf{F}_{L} \equiv e\left(\mathbf{E}+\frac{\mathbf{v}}{c} \times \mathbf{H}\right),
$$

where $\mathbf{v}$ is the electron velocity, and $\mathbf{E}$ and $\mathbf{H}$ are electric and magnetic fields of a wave.

Let us analyze the motion of a particle near the top of its trajectory (maximum of the motion amplitude), as shown in Fig. 6. Near the top of the particle trajectory one can consider electron motion as on the circumference of a circle with local gyroradius $r_{\mathrm{gyr}}$ (see Fig. 6). The time derivative of the momentum can be written using Eq. (A1) as

$$
\frac{d \mathbf{p}}{d t}=|\mathbf{p}| \frac{d \mathbf{e}_{\varphi}}{d t}=m c \gamma_{0} \frac{d \mathbf{e}_{\varphi}}{d \varphi} \frac{d \varphi}{d t}=\frac{m c^{2} \gamma_{0}}{r_{\mathrm{gyr}}} \mathbf{e}_{r},
$$

where $\mathbf{e}_{\phi}$ and $\mathbf{e}_{r}$ are local unit vectors in polar coordinates $r, \varphi$; these are shown in Fig. 6. Thus, Eq. (A2) for an ultrarelativistic particle can be rewritten as 


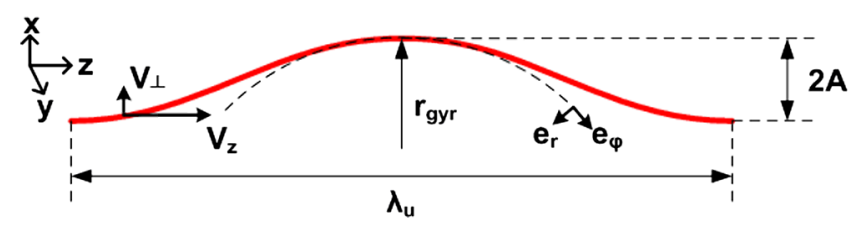

FIG. 6. Electron trajectory in the undulator.

$$
\left|\frac{d \mathbf{p}}{d t}\right|=\frac{m c^{2} \gamma_{0}}{r_{\mathrm{gyr}}}=e\left|E_{x}+H_{y}\right| .
$$

Finally, for the gyroradius we find

$$
r_{\mathrm{gyr}}=\frac{m \gamma_{0} c^{2}}{e \| E_{x}| \pm| H_{y}||},
$$

where + corresponds to the counterpropagating wave, and - corresponds to the copropagating wave. In travelingwave fields $E_{x}$ and $H_{y}$ are in phase, and these fields provide the smallest gyroradius near the top of the trajectory, when they reach maximum amplitude values. In the copropagating wave, a particle trajectory has a larger radius of curvature than in a counterpropagating wave.

To obtain formulas for the undulator $K$ parameter we use its relationship with the amplitude of wiggling $A$, as is frequently used for DC-magnet undulators [2,3], and which follows from Eq. (24) for wiggling in a traveling wave at injection phase $\varphi=0$, namely,

$$
K=\gamma_{0} A k_{u} .
$$

Geometric analysis gives the relationship between the amplitude of the oscillations and the gyroradius of the trajectory to be

$$
A=\frac{1}{r_{\mathrm{gyr}} k_{u}^{2}} .
$$

Finally, using Eqs. (A5)-(A7), we obtain

$$
K=\frac{e H_{\perp}\left(1+E_{\perp} / H_{\perp}\right)}{m c^{2}(h+k)} \equiv \frac{e H_{\perp}\left(1+E_{\perp} / H_{\perp}\right) \lambda_{u}}{2 \pi m c^{2}},
$$

where instead of $E_{x}, H_{y}$ in the general case we write amplitudes of the transverse fields $E_{\perp}, H_{\perp}$, keeping in mind that actual transverse electric and magnetic fields are to be perpendicular to one another. In a waveguide where $E_{\perp} \neq H_{\perp}$ and $h \neq k$. we reformulate Eq. (A8) for the two classes of modes. For TE modes, the relationship between transverse $E_{\perp}$ and $H_{\perp}$ fields taken at the same point is

$$
E_{\perp} / H_{\perp}=k / h .
$$

Substituting Eq. (A9) into Eq. (A8), we recover Eq. (20) for the $K$ parameter. In the TM-mode case the transverse fields are related as

$$
E_{\perp} / H_{\perp}=h / k,
$$

so we recover Eq. (21).

\section{APPENDIX B: SYSTEM OF EQUATIONS TO DESCRIBE PARTICLE MOTION AND INDIVIDUAL RADIATION SPECTRUM IN RF UNDULATOR}

Electron motion (if one does not take into account an action of the laser radiation on electron trajectory) in given electromagnetic fields can be described by vector differential equation for momentum $\mathbf{p}$ with Lorentz force in the right part of Eq. (A2). Let us introduce a new variable $\mathbf{P}$ also having a sense of a momentum:

$$
\mathbf{P}=\frac{\mathbf{p}}{\mathrm{cm}} \text {. }
$$

For this variable one can write the system of equations for each vector component as

$$
\frac{d \mathbf{P}}{d t}=\frac{e}{c m}\left(\mathbf{E}+\frac{\mathbf{v}}{c} \times \mathbf{H}\right)
$$

where $m$ is a mass of an electron in condition of the rest. This equation is supplemented with equations which connect velocity with impulse and Lorentz's gamma factor:

$$
\mathbf{v} \equiv \frac{d \mathbf{r}}{d t}=\frac{c}{\gamma} \mathbf{P}, \quad \gamma=\sqrt{1+\mathbf{P}^{2}} .
$$

Equations (B2) and (B3) define particle dynamics, if one sets initial values of velocity $\mathbf{v}(t=0)$ and initial particle coordinate $\mathbf{r}(t=0)$.

Note that Eq. (B2) can be solved in the laboratory system of coordinates $K$ as well as in a moving system of coordinates $K^{\prime}$. We prefer to calculate electron motion in the system of coordinates $K^{\prime}$. In this system a particle is not ultrarelativistic anymore. The system $K^{\prime}$ moves together electron beam with velocity of the unperturbed electron beam:

$$
v_{0} \equiv \beta_{0} c=\frac{c}{\gamma_{0}} \sqrt{\gamma_{0}^{2}-1}
$$

In this system of coordinates each electron has a condition of rest in the beginning $\vec{v}^{\prime}\left(t^{\prime} \leq 0\right)=0$; when $t^{\prime}>0$, it drifts in the negative direction of $z^{\prime}$ and simultaneously oscillates in the transverse direction until $t^{\prime} \leq T_{\max }=\frac{L}{c \gamma_{z 0}}$. If in the laboratory coordinate system a counterpropagating wave has frequency $\omega$ and propagation constant $h$, then in the system $K^{\prime}$ the electron entered in the interaction section sees a counterpropagating wave with the higher frequency:

$$
\omega^{\prime}=\left(\omega+h v_{0}\right) \gamma_{0}
$$

and the higher longitudinal propagation constant:

$$
h_{z}^{\prime}=\left(h+\beta_{0} \frac{\omega}{c}\right) \gamma_{0} .
$$


Formally both systems $K$ and $K^{\prime}$ are equivalent, but calculations of electron trajectories and radiation spectra have shown that accuracy of calculations is higher in the moving system $K^{\prime}$.

The intensity spectrum of the forward waves radiated by an oscillating electron in the $z$ direction is given in the laboratory system of coordinates by the equation [14]

$\hat{I} \equiv \frac{d^{2} I}{d \Omega d \omega}=I_{0} \omega^{2}\left|\int_{0}^{L} \beta_{\perp} \exp \left[i \omega\left(\frac{z}{c}-t\right)\right] \frac{d z}{\beta_{z}}\right|^{2}$,

where $I_{0}$ is a multiplier consisted of the fundamental constants only. For spectrum calculations in the moving system of coordinates $K^{\prime}$ let us simplify Eq. (B7) substituting the integration along $z$ by integration on time:

$$
\hat{I}^{\prime}\left(\omega^{\prime}\right)=I_{0}^{\prime} \omega^{\prime 2}\left|\int_{0}^{T_{\max }} \nu_{\perp}\left(t^{\prime}\right) \exp \left[i \omega^{\prime}\left(\frac{z^{\prime}\left(t^{\prime}\right)}{c}-t^{\prime}\right)\right] d t^{\prime}\right|^{2} .
$$

Because a radiated plane wave, propagated along axis $z^{\prime}$ with frequency $\omega^{\prime}$, has in the laboratory system of coordinates the frequency $\omega^{\prime \prime}$,

$$
\omega^{\prime \prime}=\omega^{\prime}\left(1+\beta_{0}\right) \gamma_{0},
$$

the form of the radiation spectrum in the laboratory system of coordinates $\hat{I}\left(\omega^{\prime \prime}\right)$ can be easily calculated by means of $\hat{I}^{\prime}\left(\omega^{\prime}\right)$ using the formula

$$
\hat{I}\left(\omega^{\prime \prime}\right)=\hat{I}^{\prime}\left(\omega^{\prime}=\frac{\omega^{\prime \prime}}{\left(1+\beta_{0}\right) \gamma_{0}}\right) .
$$

[1] J. M. J. Madey, J. Appl. Phys. 42, 1906 (1971).

[2] Zh. Huang and K.-J. Kim, Phys. Rev. ST Accel. Beams 10, 034801 (2007).

[3] M. Seidel, DESY-TESLA-FEL Report No. 2001-08, 2001 [http://tesla.desy.de/new_pages/FEL_Reports/2001/ pdf_files/fel2001-08.pdf].

[4] J. Pflueger, B. Faatz, M. Tischer, and T. Vielit, Nucl. Instrum. Methods Phys. Res., Sect. A 507, 186 (2003).

[5] W. M. Fawley, Zh. Huang, K.-J. Kim, and N. A. Vinokurov, Nucl. Instrum. Methods Phys. Res., Sect. A 483, 537 (2002).
[6] T. Shintake, K. Huke, J. Tanaka, I. Sato, and I. Kumabe, Jpn. J. Appl. Phys. 22, 844 (1983).

[7] G. G. Denisov, A. V. Smorgonsky, V.P. Gubanov, S. D. Korovin, V. V. Rostov, and M. I. Yalandin, Int. J. Infrared Millim. Waves 5, 1389 (1984).

[8] Sami G. Tantawi, CLIC Workshop 2013, High Gradient Session, CERN, 2013 (https://indico.cern .ch/contributionDisplay.py? contribId $=41 \&$ sessionId $=10$ \&confId=204269).

[9] C. Pellegrini, in Proceedings of the 27th International Free Electron Laser Conference, Stanford, CA, 2005, p. 203 [http://accelconf.web.cern.ch/AccelConf/f05/ PAPERS/MOPP060.PDF].

[10] N. I. Zaitsev, E. V. Ilyakov, S. V. Kuzikov, I. S. Kulagin, V. K. Lygin, M. A. Moiseev, M. I. Petelin, and A. S. Shevchenko, Radiophys. Quantum Electron. 48, 737 (2005).

[11] M. A. LaPointe, J. L. Hirshfield, E. V. Kozyrev, O. A. Nezhevenko, S. V. Shchelkunov, and V.P. Yakovlev, in Proceedings of the Infrared Millimeter Waves and 14th International Conference on Teraherz Electronics, Shanghai, China, 2006 (IEEE, New York, 2006), p. 127.

[12] A. V. Gaponov and M. A. Miller, Zh. Eksp. Teor. Fiz. 34, 242 (1958).

[13] L. D. Landau, E. M. Lifshitz, and L.P. Pitaevskii, Electrodynamics of Continuous Media (ButterworthHeinemann, MA, 1984), Vol. 8, 2nd ed.

[14] T. M. Tran, B. G. Danly, and J.S. Wurtele, IEEE J. Quantum Electron. 23, 1578 (1987).

[15] C. Chang, M. Shumail, S. Tantawi, J. Neilson, and C. Pellegrini, Appl. Phys. Lett. 101, 161102 (2012).

[16] P. J. Eecen, T. J. Schep, and A. V. Tulupov, Phys. Rev. E 52, 5460 (1995).

[17] S. V. Kuzikov, A. A. Vikharev, M.E. Plotkin, J. L. Hirshfield, T.C. Marshall, G. V. Sotnikov, and A. V. Peskov, in Proceedings of the 2nd International Particle Accelerator Conference, San Sebastián, Spain (EPS-AG, Spain, 2011), THPC169, pp. 3293-3295 [http://accelconf.web.cern.ch/AccelConf/IPAC2011/papers/ thpc169.pdf].

[18] S. V. Kuzikov, J. L. Hirshfield, Y. Jiang, T. C. Marshall, and A. A. Vikharev, in Proceedings of the 15th Advanced Accelerator Concepts Workshop, 2012, Austin, Texas, USA, edited by R. Zgadzaj, E. Gaul, and M. C. Downer, AIP Conf. Proc. No. 1507 (AIP, New York, 2012), pp. 458-463 [http://dx.doi.org/10.1063/1.4773740]. 